
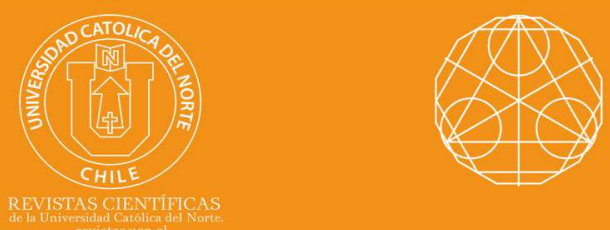

\title{
Strongly convexity on fractal sets and some inequalitiess
}

Rainier V. Sánchez C. ${ }^{1}$ (D) orcid.org/0000-0002-6739-5102

José E. Sanabria ${ }^{2}$ @i orcid.org/0000-0002-9749-4099

${ }^{1}$ Instituto Superior de Formación Docente Salomé Ureña, Dept. de Matemáticas, Santo Domingo, República Dominicana.

rainiersan76@gmail.com

${ }^{2}$ Universidad de Sucre, Dept. de Matemáticas, Sincelejo, Colombia.

曰jesanabri@gmail.com

Received: November 2018 | Accepted: November 2019

\section{Abstract:}

We introduce a generalization of the concept of a strongly convex function on a fractal set, study some algebraic properties and establish Jensen-type and Hermite-Hadamard-type inequalities.

Keywords: Convex function; Generalized convex function; Strongly convex function; Fractal set.

MSC (2010): 26D07; 26D15; 26A51; 39B62.

\section{Cite this article as (IEEE citation style):}

R. V. Sánchez C. and J. E. Sanabria, "Strongly convexity on

fractal sets and some inequalities", Proyecciones (Antofagasta, On line), vol. 39, no. 1, pp. 01-13, Feb. 2020, doi: 10.22199/issn.0717-6279-2020-01-0001. [Accessed dd-mm-yyyy].

Article copyright: (c) 2020 Rainier V. Sánchez C. and José E. Sanabria. This is an open access article distributed under the terms of the Creative Commons Licence, which permits unrestricted use and distribution provided the original author and source are credited.

(cc) BY 


\section{Introduction and preliminaries}

The concept of convex function and its generalizations have been the subject of numerous studies that have provided interesting results in some branches related to mathematics, such as geometric functional analysis, mathematical economics, convex analysis, nonlinear optimization, linear programming, theory of control and dynamic systems. On the other hand, the concept of local fractional calculus (also called fractal calculus) introduced by Kolwankar and Gangal [3] has received considerable attention for its application in non-differentiable problems of science and engineering. Motivated by these applications, in 2012, Yang [11] established the analysis of local fractional functions on fractal sets systematically, which included local fractional calculus and the monotonicity of functions. Recently, the fractal calculus has been used by Mo, Sui and $\mathrm{Yu}[6]$ to introduce a generalization of the concept of convex function on fractal sets and to establish inequalities of Jensen and Hermite-Hadamard for generalized convex functions. Similarly, Sun [10] introduced the concept of generalized harmonically convex function on fractal sets and established the respective Hermite-Hadamard inequalities for this class of functions. This work continues in the same line of investigation of the two works mentioned above, but in our case we introduce a generalization of the concept of a strongly convex function on a fractal set, study some algebraic properties and establish Jensen-type and Hermite-Hadamard-type inequalities.

Strongly convex functions have been introduced by Polyak in 1966 [8] and has been studied and generalized by different authors $[1,4,5,9]$. Recall the definition of this class of functions.

Definition 1.1. Let $I \subset \mathbf{R}$ be an interval and $c$ be a positive real number. A function $f: I \rightarrow \mathbf{R}$ is said to be strongly convex with modulus $c$ if

$$
f(t x+(1-t) y) \leq t f(x)+(1-t) f(y)-c t(1-t)(x-y)^{2},
$$

for all $x, y \in I$ and $t \in[0,1]$.

Strongly convex functions have been used for proving the convergence of a gradient type algorithm for minimizing a function. They play an important role in optimization theory and mathematical economics. [7, 9]

Recenty, the theory of Yang's fractional sets [11] was introduced as follows. For $0<\alpha \leq 1$, we have following $\alpha$-type set of element sets:

$\mathbf{Z}^{\alpha}=\left\{0^{\alpha}, \pm 1^{\alpha}, \pm 2^{\alpha}, \ldots, \pm n^{\alpha}, \ldots\right\}$ (integer numbers $\alpha$-type). 


$$
\begin{array}{ll}
\mathbf{Q}^{\alpha}=\left\{m^{\alpha}=\left(\frac{p}{q}\right)^{\alpha}: p, q \in \mathbf{Z}, q \neq 0\right\} & \text { (rationals numbers } \alpha \text {-type) } . \\
\mathbf{J}^{\alpha}=\left\{m^{\alpha} \neq\left(\frac{p}{q}\right)^{\alpha}: p, q \in \mathbf{Z}, q \neq 0\right\} & \text { (irrational numbers } \alpha \text {-type) } . \\
\mathbf{R}^{\alpha}=\mathbf{Q}^{\alpha} \cup \mathbf{J}^{\alpha} & \text { (real line numbers } \alpha \text {-type). }
\end{array}
$$

We call fractal set to $\mathbf{R}^{\alpha}$ and any subset of it. The following facts are found in [2], [11] and [12].

If $a^{\alpha}, b^{\alpha}$ and $c^{\alpha}$ belong to the set $\mathbf{R}^{\alpha}$ of real line numbers, then one has the following:

1. $a^{\alpha}+b^{\alpha}$ and $a^{\alpha} b^{\alpha}$ belong to the set $\mathbf{R}^{\alpha}$.

2. $a^{\alpha}+b^{\alpha}=b^{\alpha}+a^{\alpha}=(a+b)^{\alpha}=(b+a)^{\alpha}$.

3. $a^{\alpha}+\left(b^{\alpha}+c^{\alpha}\right)=\left(a^{\alpha}+b^{\alpha}\right)+c^{\alpha}$.

4. $a^{\alpha} b^{\alpha}=b^{\alpha} a^{\alpha}=(a b)^{\alpha}=(b a)^{\alpha}$.

5. $a^{\alpha}\left(b^{\alpha} c^{\alpha}\right)=\left(a^{\alpha} b^{\alpha}\right) c^{\alpha}$.

6. $a^{\alpha}\left(b^{\alpha}+c^{\alpha}\right)=a^{\alpha} b^{\alpha}+a^{\alpha} c^{\alpha}$.

7. $a^{\alpha}+0^{\alpha}=0^{\alpha}+a^{\alpha}=a^{\alpha}$ y $a^{\alpha} 1^{\alpha}=1^{\alpha} a^{\alpha}=a^{\alpha}$.

It is important to note that in this theory the number $\left(a^{2}\right)^{\alpha} \in \mathbf{R}^{\alpha}$ will be represented by $a^{2 \alpha}$.

Now we introduce some basic definitions about the local factional calculus.

Definition 1.2. [11] A non-differentiable function $f: \mathbf{R} \rightarrow \mathbf{R}^{\alpha}, x \rightarrow f(x)$ is called local fractional continuous at $x_{0}$, if for any $\varepsilon>0$, there exists $\delta>0$, such that

$$
\left|f(x)-f\left(x_{0}\right)\right|<\varepsilon
$$

holds for $\left|x-x_{0}\right|<\delta$, where $\varepsilon, \delta \in \mathbf{R}$. If a function $f$ is local fractional continuous on an interval $I$, we denote $f \in C_{\alpha}(I)$.

Definition 1.3. [11] The local fractional derivative of $f(x)$ of order $\alpha$ at $x=x_{0}$ is defined by

$$
f^{(\alpha)}\left(x_{0}\right)=\left.\frac{d^{\alpha} f(x)}{d x^{\alpha}}\right|_{x=x_{0}}=\lim _{x \rightarrow x_{0}} \frac{\Delta^{\alpha}\left(f(x)-f\left(x_{0}\right)\right)}{\left(x-x_{0}\right)^{\alpha}}
$$

where $\Delta^{\alpha}\left(f(x)-f\left(x_{0}\right)\right) \cong \Gamma(1+\alpha)\left(f(x)-f\left(x_{0}\right)\right)$ and $\Gamma$ is the familiar Gamma function. 
Let $f^{(\alpha)}(x)=D_{x}^{\alpha} f(x)$. If there exists $f^{((k+1) \alpha)}(x)=\overbrace{D_{x}^{\alpha} \cdots D_{x}^{\alpha}}^{k+1 \text { times }} f(x)$ for any $x \in I \subseteq \mathbf{R}$, then we denote $f \in D_{(k+1) \alpha}(I)$, where $k=0,1,2, \ldots$.

Definition 1.4. [11] Let $f \in C_{\alpha}[a, b]$. The local fractional integral of $f$ on the interval $[a, b]$ of order $\alpha$ (denoted by ${ }_{a} I_{b}^{(\alpha)} f$ ) is defined by

$$
{ }_{a} I_{b}^{(\alpha)} f(t)=\frac{1}{\Gamma(1+\alpha)} \int_{a}^{b} f(t)(d t)^{\alpha}=\frac{1}{\Gamma(1+\alpha)} \lim _{\Delta t \rightarrow 0} \sum_{j=0}^{N-1} f\left(t_{j}\right)\left(\Delta t_{j}\right)^{\alpha},
$$

with $\Delta t=\max \left\{\Delta t_{0}, \Delta t_{1}, \ldots, \Delta t_{N-1}\right\}$ and $\Delta t_{j}=t_{j+1}-t_{j}$ for $j=0,1, \ldots$, $N-1$, where $a=t_{0}<t_{1}<\cdots<t_{i}<\cdots<t_{N-1}<t_{N}=b$ is a partition of the interval $[a, b]$.

Here, it follows that ${ }_{a} I_{b}^{(\alpha)} f=0$ if $a=b$ and ${ }_{a} I_{b}^{(\alpha)} f=-{ }_{b} I_{a}^{(\alpha)} f$ if $a<b$. If ${ }_{a} I_{x}^{(\alpha)} f$ there exits for any $x \in[a, b]$, then it is denoted by $f \in I_{x}^{(\alpha)}[a, b]$.

In 2014, H. Mo et al. [6] used the local fractional calculus to introduce the following generalized convex function.

Definition 1.5. [6] Let $f: I \rightarrow \mathbf{R}^{\alpha}$. For any $x, y \in I$ and $t \in[0,1]$, if the following inequality

$$
f(t x+(1-t) y) \leq t^{\alpha} f(x)+(1-t)^{\alpha} f(y),
$$

holds, then $f$ is called a generalized convex function on $I$.

We will denote by $G C_{\alpha}(I)$ to the set of the generalized convex functions on $I$, that is to say,

$$
G C_{\alpha}(I)=\left\{f: I \rightarrow \mathbf{R}^{\alpha} \mid f \text { is a generalized convex function on } I\right\} .
$$

\section{Main Results}

In this section we introduce the concept of a strongly convex generalized function on a fractal set with modulo $c$, study some algebraic properties and establish Jensen-type and Hermite-Hadamard-type inequalities.

Definition 2.1. Let $I \subset \mathbf{R}$ be an interval and $c \in \mathbf{R}_{+}$. A function $f: I \rightarrow$ $\mathbf{R}^{\alpha}$ is called generalized strongly convex with modulus $c$ if

$$
f(t x+(1-t) y) \leq t^{\alpha} f(x)+(1-t)^{\alpha} f(y)-c^{\alpha} t^{\alpha}(1-t)^{\alpha}(x-y)^{2 \alpha},
$$

for all $x, y \in I$ and $t \in[0,1]$. 
Remark 2.2. Note that for particular cases of the numbers $0<\alpha \leq 1$ and $c \in \mathbf{R}_{+}$, we recover well known classical concepts of convex functions as is shown.

1. If $\alpha=1$ then the generalized strongly convex functions are the strongly convex functions.

2. If $c=0$ then the generalized strongly convex functions are the generalized convex functions.

3. If $\alpha=1$ and $c=0$ then the generalized strongly convex functions are the convex functions.

The family of all generalized strongly convex functions with modulus $c$ is denoted by $G S C_{\alpha}^{c}(I)$; that is,

$G S C_{\alpha}^{c}(I)=\left\{f: I \rightarrow \mathbf{R}^{\alpha} \mid f\right.$ is generalized strongly convex with modulus $\left.c\right\}$.

Note that if $f \in G S C_{\alpha}^{c}(I)$ and $f(0)=0^{\alpha}$ then $f(t x) \leq t^{\alpha} f(x)$. Also, if $f \in G S C_{\alpha}^{c}(I)$ then $f\left(\frac{x+y}{2}\right) \leq \frac{f(x)+f(y)}{2^{\alpha}}-c^{\alpha}\left(\frac{x-y}{2}\right)^{2 \alpha}$.

Theorem 2.3. If $f, g \in G S C_{\alpha}^{\frac{c}{2}}(I)$, then $f+g \in G S C_{\alpha}^{c}(I)$.

Proof. Let $f, g \in G S C C_{\alpha}^{\frac{c}{2}}(I), c>0, x, y \in I$ and $t \in[0,1]$. Then

$$
\begin{aligned}
(f+g)(t x+(1-t) y)= & f(t x+(1-t) y)+g(t x+(1-t) y) \\
\leq & t^{\alpha} f(x)+(1-t)^{\alpha} f(y)-\frac{c^{\alpha}}{2^{\alpha}} t^{\alpha}(1-t)^{\alpha}(x-y)^{2 \alpha} \\
& +t^{\alpha} g(x)+(1-t)^{\alpha} g(y)-\frac{c^{\alpha}}{2^{\alpha}} t^{\alpha}(1-t)^{\alpha}(x-y)^{2 \alpha} \\
= & t^{\alpha}(f(x)+g(x))+(1-t)^{\alpha}(f(y)+g(y)) \\
& -2^{\alpha} \frac{c^{\alpha}}{2^{\alpha}} t^{\alpha}(1-t)^{\alpha}(x-y)^{2 \alpha} \\
= & t^{\alpha}(f+g)(x)+(1-t)^{\alpha}(f+g)(y) \\
& -c^{\alpha} t^{\alpha}(1-t)^{\alpha}(x-y)^{2 \alpha} .
\end{aligned}
$$

So $f+g \in G S C_{\alpha}^{c}(I)$.

Theorem 2.4. If $f \in G S C_{\alpha}^{\frac{c}{\lambda}}(I)$, then $\lambda^{\alpha} f \in G S C_{\alpha}^{c}(I)$, when $\lambda \in \mathbf{R}^{+}$. 
Proof. If $x, y \in I$ and $t \in[0,1]$, then

$$
\begin{aligned}
\left(\lambda^{\alpha} f\right)(t x+(1-t) y)= & \lambda^{\alpha} f(t x+(1-t) y) \\
\leq & \lambda^{\alpha}\left[t^{\alpha} f(x)+(1-t)^{\alpha} f(y)-\frac{c^{\alpha}}{\lambda^{\alpha}} t^{\alpha}(1-t)^{\alpha}(x-y)^{2 \alpha}\right] \\
= & t^{\alpha} \lambda^{\alpha} f(x)+(1-t)^{\alpha} \lambda^{\alpha} f(y)-c^{\alpha} t^{\alpha}(1-t)^{\alpha}(x-y)^{2 \alpha} \\
= & t^{\alpha}\left(\lambda^{\alpha} f\right)(x)+(1-t)^{\alpha}\left(\lambda^{\alpha} f\right)(y) \\
& -c^{\alpha} t^{\alpha}(1-t)^{\alpha}(x-y)^{2 \alpha},
\end{aligned}
$$

and hence $\lambda^{\alpha} f \in G S C_{\alpha}^{c}(I)$.

Theorem 2.5. If $f_{n}: I \longrightarrow \mathbf{R}^{\alpha}, n \in \mathbf{N}$, is a sequence of generalized strongly convex functions with modulus $c$ converging pointwise to a function $f: I \longrightarrow \mathbf{R}^{\alpha}$, then $f \in G S C_{\alpha}^{c}(I)$.

Proof. Let $x, y \in I, t \in[0,1]$ and $\lim _{n \rightarrow \infty} f_{n}(x)=f(x)$, then

$$
\begin{aligned}
f(t x+(1-t) y)= & \lim _{n \rightarrow \infty} f_{n}(t x+(1-t) y) \\
\leq & \lim _{n \rightarrow \infty}\left(t^{\alpha} f_{n}(x)+(1-t)^{\alpha} f_{n}(y)-c^{\alpha} t^{\alpha}(1-t)^{\alpha}(x-y)^{2 \alpha}\right) \\
= & t^{\alpha} \lim _{n \rightarrow \infty} f_{n}(x)+(1-t)^{\alpha} \lim _{n \rightarrow \infty} f_{n}(y) \\
& -\lim _{n \rightarrow \infty} c^{\alpha} t^{\alpha}(1-t)^{\alpha}(x-y)^{2 \alpha} \\
= & t^{\alpha} f(x)+(1-t)^{\alpha} f(y)-c^{\alpha} t^{\alpha}(1-t)^{\alpha}(x-y)^{2 \alpha}
\end{aligned}
$$

that is, $f \in G S C_{\alpha}^{c}(I)$.

Remark 2.6. If $x, y \in I, t \in[0,1]$ and $\bar{x}=t x+(1-t) y$, then we have

$$
t^{\alpha}(1-t)^{\alpha}(x-y)^{2 \alpha}=t^{\alpha}(x-\bar{x})^{\alpha}+(1-t)^{\alpha}(y-\bar{x})^{\alpha} .
$$

Hence, a function $f: I \rightarrow \mathbf{R}^{\alpha}$ is generalized strongly convex with modulus $c$ if and only if

$f(t x+(1-t) y) \leq t^{\alpha} f(x)+(1-t)^{\alpha} f(y)-c^{\alpha} t^{\alpha}(x-\bar{x})^{\alpha}+(1-t)^{\alpha}(y-\bar{x})^{\alpha}$,

for all $x, y \in I$ and $t \in[0,1]$. This fact serve as motivation to establish a generalization of the version of the discrete Jensen-type inequality given in [5]. 
Theorem 2.7 (generalized Jensen-type inequality). Assume that $f \in$ $G S C_{\alpha}^{c}(I)$. Then for any $x_{i} \in I$ and $t_{i} \in[0,1](i=1,2, \ldots, n)$ with $\sum_{i=1}^{n} t_{i}=1$ and $\bar{x}=\sum_{i=1}^{n} t_{i} x_{i}$, we have

$$
f\left(\sum_{i=1}^{n} t_{i} x_{i}\right) \leq \sum_{i=1}^{n} t_{i}^{\alpha} f\left(x_{i}\right)-c^{\alpha} \sum_{i=1}^{n} t_{i}^{\alpha}\left(x_{i}-\bar{x}\right)^{2 \alpha} .
$$

Proof. The proof is by induction on $n$. When $n=2$, we have

$$
\begin{aligned}
f\left(\sum_{i=1}^{2} t_{i} x_{i}\right) & =f\left(t_{1} x_{1}+t_{2} x_{2}\right)=f\left(t_{1} x_{1}+\left(1-t_{1}\right) x_{2}\right) \\
& \leq t_{1}^{\alpha} f\left(x_{2}\right)+\left(1-t_{1}\right)^{\alpha} f\left(x_{2}\right)-c^{\alpha}\left(t_{1}^{\alpha}\left(x_{1}-\bar{x}\right)^{2 \alpha}+\left(1-t_{1}\right)^{\alpha}\left(x_{2}-\bar{x}\right)^{2 \alpha}\right) \\
& =t_{1}^{\alpha} f\left(x_{2}\right)+\left(1-t_{1}\right)^{\alpha} f\left(x_{2}\right)-c^{\alpha}\left(t_{1}^{\alpha}\left(x_{1}-\bar{x}\right)^{2 \alpha}+t_{2}^{\alpha}\left(x_{2}-\bar{x}\right)^{2 \alpha}\right) \\
& =\sum_{i=1}^{2} t_{i}^{\alpha} f\left(x_{i}\right)-c^{\alpha} \sum_{i=1}^{2} t_{i}^{\alpha}\left(x_{i}-\bar{x}\right)^{2 \alpha}
\end{aligned}
$$

where $\bar{x}=\sum_{i=1}^{2} t_{i} x_{i}$.

Assume that for $n=k$ the inequality is also true, i.e. for any $x_{1}, x_{2}, \ldots, x_{k} \in$ $I$ and $t_{i} \in[0,1](i=1,2, \ldots, k)$ with $\sum_{i=1}^{k} t_{i}=1$, we have

$$
f\left(\sum_{i=1}^{k} t_{i} x_{i}\right) \leq \sum_{i=1}^{k} t_{i}^{\alpha} f\left(x_{i}\right)-c^{\alpha} \sum_{i=1}^{k} t_{i}^{\alpha}\left(x_{i}-\bar{x}\right)^{2 \alpha}
$$

where $\bar{x}=\sum_{i=1}^{k} t_{i} x_{i}$.

Now let us verify that for $n=k+1$ the inequality is true. If $x_{1}, x_{2}, \ldots, x_{k}$, $x_{k+1} \in I$ and $t_{i} \in[0,1](i=1,2, \ldots, k, k+1)$ with $\sum_{i=1}^{k+1} t_{i}=1$, then let $\bar{x}=\sum_{i=1}^{k+1} t_{i} x_{i}$ and $\bar{y}=\sum_{i=1}^{k} \lambda_{i} x_{i}$, where $\lambda_{i}=\frac{t_{i}}{1-t_{k+1}}$. By the generalized strongly convexity of $f$, we have 


$$
\begin{aligned}
f\left(\sum_{i=1}^{k+1} t_{i} x_{i}\right)= & f\left(t_{k+1} x_{k+1}+\left(1-t_{k+1}\right) \bar{y}\right) \\
\leq & t_{k+1}^{\alpha} f\left(x_{k+1}\right)+\left(1-t_{k+1}\right)^{\alpha} f(\bar{y}) \\
& -c^{\alpha}\left[t_{k+1}^{\alpha}\left(x_{k+1}-\bar{x}\right)^{2 \alpha}+\left(1-t_{k+1}\right)^{\alpha}(\bar{y}-\bar{x})^{2 \alpha}\right]
\end{aligned}
$$

Since $\sum_{i=1}^{k} \lambda_{i}=1$, then by inductive hypothesis it follows that

$$
\begin{aligned}
f\left(\sum_{i=1}^{k+1} t_{i} x_{i}\right) \leq & t_{k+1}^{\alpha} f\left(x_{k+1}\right)+\left(1-t_{k+1}\right)^{\alpha}\left[\sum_{i=1}^{k} \lambda_{i}^{\alpha} f\left(x_{i}\right)-c^{\alpha} \sum_{i=1}^{k} \lambda_{i}^{\alpha}\left(x_{i}-\bar{y}\right)^{2 \alpha}\right] \\
& -c^{\alpha}\left[t_{k+1}^{\alpha}\left(x_{k+1}-\bar{x}\right)^{2 \alpha}+\left(1-t_{k+1}\right)^{\alpha}(\bar{y}-\bar{x})^{2 \alpha}\right] \\
= & t_{k+1}^{\alpha} f\left(x_{k+1}\right)+\sum_{i=1}^{k} t_{i}^{\alpha} f\left(x_{i}\right)-c^{\alpha} \sum_{i=1}^{k} t_{i}^{\alpha}\left(x_{i}-\bar{y}\right)^{2 \alpha} \\
& -c^{\alpha} t_{k+1}^{\alpha}\left(x_{k+1}-\bar{x}\right)^{2 \alpha}-c^{\alpha}\left(\sum_{i=1}^{k} t_{i}\right)^{\alpha}(\bar{y}-\bar{x})^{2 \alpha} \\
= & \sum_{i=1}^{k+1} t_{i}^{\alpha} f\left(x_{i}\right)-c^{\alpha} \sum_{i=1}^{k} t_{i}^{\alpha}\left[\left(x_{i}-\bar{y}\right)^{2 \alpha}+(\bar{y}-\bar{x})^{2 \alpha}\right] \\
& -c^{\alpha} t_{k+1}^{\alpha}\left(x_{k+1}-\bar{x}\right)^{2 \alpha} \\
= & \sum_{i=1}^{k+1} t_{i}^{\alpha} f\left(x_{i}\right)-c^{\alpha} \sum_{i=1}^{k} t_{i}^{\alpha}\left[\left(x_{i}-\bar{x}\right)^{2 \alpha}-2^{\alpha}\left(x_{i}-\bar{y}\right)^{\alpha}(\bar{y}-\bar{x})^{\alpha}\right] \\
& -c^{\alpha} t_{k+1}^{\alpha}\left(x_{k+1}-\bar{x}\right)^{2 \alpha} \\
= & \sum_{i=1}^{k+1} t_{i}^{\alpha} f\left(x_{i}\right)-c^{\alpha} \sum_{i=1}^{k} t_{i}^{\alpha}\left[\left(x_{i}-\bar{x}\right)^{2 \alpha}-2^{\alpha}\left(x_{i}-\bar{y}\right)^{\alpha} \frac{t_{k+1}^{\alpha}}{\left(1-t_{k+1}\right)^{\alpha}}\right. \\
& \left.\left(\bar{x}-x_{k+1}\right)^{\alpha}\right]-c^{\alpha} t_{k+1}^{\alpha}\left(x_{k+1}-\bar{x}\right)^{2 \alpha} \\
& \sum_{i=1}^{k+1} t_{i}^{\alpha} f\left(x_{i}\right)-c^{\alpha} \sum_{i=1}^{k} t_{i}^{\alpha}\left(x_{i}-\bar{x}\right)^{2 \alpha}-c^{\alpha} t_{k+1}^{\alpha}\left(x_{k+1}-\bar{x}\right)^{2 \alpha} t_{k+1}^{\alpha}\left(\bar{x}-x_{k+1}\right)^{\alpha} \sum_{i=1}^{k} \lambda_{i}^{\alpha}\left(x_{i}-\bar{y}\right)^{\alpha} \\
& \sum_{i=1}^{k+1} t_{i}^{\alpha} f\left(x_{i}\right)-c^{\alpha} \sum_{i=1}^{k} t_{i}^{\alpha}\left(x_{i}-\bar{x}\right)^{2 \alpha} \\
= & \\
&
\end{aligned}
$$




$$
\begin{aligned}
& +2^{\alpha} c^{\alpha} t_{k+1}^{\alpha}\left(\bar{x}-x_{k+1}\right)^{\alpha}\left[\left(\sum_{i=1}^{k} \lambda_{i} x_{i}\right)^{\alpha}-\bar{y}^{\alpha} \sum_{i=1}^{k} \lambda_{i}^{\alpha}\right] \\
= & \sum_{i=1}^{k+1} t_{i}^{\alpha} f\left(x_{i}\right)-c^{\alpha} \sum_{i=1}^{k+1} t_{i}^{\alpha}\left(x_{i}-\bar{x}\right)^{2 \alpha}+2^{\alpha} c^{\alpha} t_{k+1}^{\alpha}\left(\bar{x}-x_{k+1}\right)^{\alpha}\left(\bar{y}^{\alpha}-\bar{y}^{\alpha} 1^{\alpha}\right) \\
= & \sum_{i=1}^{k+1} t_{i}^{\alpha} f\left(x_{i}\right)-c^{\alpha} \sum_{i=1}^{k+1} t_{i}^{\alpha}\left(x_{i}-\bar{x}\right)^{2 \alpha} .
\end{aligned}
$$

Hence the inequality holds for $\mathrm{n}=\mathrm{k}+1$, and thus for all $n$.

Theorem 2.8. A function $f: I \rightarrow \mathbf{R}^{\alpha}$ is generalized strongly convex with modulus $c$ if and only if the function $g: I \rightarrow \mathbf{R}^{\alpha}$ defined by $g(x)=$ $f(x)-c^{\alpha} x^{2 \alpha}$ is generalized convex.

Proof. Suppose that $f \in G S C_{\alpha}^{c}(I)$, then

$$
\begin{aligned}
g(t x+(1-t) y)= & f(t x+(1-t) y)-c^{\alpha}(t x+(1-t) y)^{2 \alpha} \\
\leq & t^{\alpha} f(x)+(1-t)^{\alpha} f(y)-c^{\alpha} t^{\alpha}(1-t)^{\alpha}(x-y)^{2 \alpha}-c^{\alpha}(t x+(1-t) y)^{2 \alpha} \\
= & t^{\alpha} f(x)+(1-t)^{\alpha} f(y)-c^{\alpha} t^{\alpha}(1-t)^{\alpha}\left(x^{2 \alpha}-2^{\alpha} x^{\alpha} y^{\alpha}+y^{2 \alpha}\right) \\
& -c^{\alpha}\left(t^{2 \alpha} x^{2 \alpha}+2^{\alpha} t^{\alpha} x^{\alpha}(1-t)^{\alpha} y^{\alpha}+(1-t)^{2 \alpha} y^{2 \alpha}\right) \\
= & t^{\alpha} f(x)+(1-t)^{\alpha} f(y)-c^{\alpha} t^{\alpha}\left(x^{2 \alpha}-2^{\alpha} x^{\alpha} y^{\alpha}+y^{2 \alpha}-t^{\alpha} x^{2 \alpha}+2^{\alpha} t^{\alpha} x^{\alpha} y^{\alpha}\right. \\
& \left.-t^{\alpha} y^{2 \alpha}\right)-c^{\alpha}\left(t^{2 \alpha} x^{2 \alpha}+2^{\alpha} t^{\alpha} x^{\alpha} y^{\alpha}-2^{\alpha} t^{2 \alpha} x^{\alpha} y^{\alpha}+y^{2 \alpha}-2^{\alpha} t^{\alpha} y^{2 \alpha}+t^{2 \alpha} y^{2 \alpha}\right) \\
= & t^{\alpha} f(x)+(1-t)^{\alpha} f(y)-c^{\alpha} t^{\alpha} x^{2 \alpha}+2^{\alpha} c^{\alpha} t^{\alpha} x^{\alpha} y^{\alpha}-c^{\alpha} t^{\alpha} y^{2 \alpha}+c^{\alpha} t^{2 \alpha} x^{2 \alpha} \\
& -2^{\alpha} c^{\alpha} t^{2 \alpha} x^{\alpha} y^{\alpha}+c^{\alpha} t^{2 \alpha} y^{2 \alpha}-c^{\alpha} t^{2 \alpha} x^{2 \alpha}-2^{\alpha} c^{\alpha} t^{\alpha} x^{\alpha} y^{\alpha}+2^{\alpha} c^{\alpha} t^{2 \alpha} x^{\alpha} y^{\alpha} \\
& -c^{\alpha} y^{2 \alpha}+2^{\alpha} c^{\alpha} t^{\alpha} y^{2 \alpha}-c^{\alpha} t^{2 \alpha} y^{2 \alpha} \\
= & \left(t^{\alpha} f(x)-c^{\alpha} t^{\alpha} x^{2 \alpha}\right)+(1-t)^{\alpha} f(y)-c^{\alpha} t^{\alpha} y^{2 \alpha}-c^{\alpha} y^{2 \alpha}+2^{\alpha} c^{\alpha} t^{\alpha} y^{2 \alpha} \\
= & t^{\alpha}\left(f(x)-c^{\alpha} x^{2 \alpha}\right)+(1-t)^{\alpha} f(y)-c^{\alpha} y^{2 \alpha}+c^{\alpha} t^{\alpha} y^{2 \alpha} \\
= & t^{\alpha}\left(f(x)-c^{\alpha} x^{2 \alpha}\right)+(1-t)^{\alpha} f(y)-(1-t)^{\alpha} c^{\alpha} y^{2 \alpha} \\
= & t^{\alpha}\left(f(x)-c^{\alpha} x^{2 \alpha}\right)+(1-t)^{\alpha}\left(f(y)-c^{\alpha} y^{2 \alpha}\right) \\
= & t^{\alpha} g(x)+(1-t)^{\alpha} g(y),
\end{aligned}
$$

for all $x, y \in I$ and $t \in[0,1]$. This shows that $g \in G C_{\alpha}^{c}(I)$. 
Conversely, assume that $g \in G C_{\alpha}^{c}(I)$. Then

$$
g(t x+(1-t) y) \leq t^{\alpha} g(x)+(1-t)^{\alpha} g(y),
$$

for all $x, y \in I$ and $t \in[0,1]$; that is,

$f(t x+(1-t) y)-c^{\alpha}(t x+(1-t) y)^{2 \alpha} \leq t^{\alpha}\left(f(x)-c^{\alpha} x^{2 \alpha}\right)+(1-t)^{\alpha}\left(f(y)-c^{\alpha} y^{2 \alpha}\right)$,

for all $x, y \in I$ and $t \in[0,1]$. From the above formula it follows that

$$
\begin{aligned}
f(t x+(1-t) y) \leq & t^{\alpha} f(x)-c^{\alpha} t^{\alpha} x^{2 \alpha}+(1-t)^{\alpha} f(y)-c^{\alpha}(1-t)^{\alpha} y^{2 \alpha} \\
& +c^{\alpha}\left(t^{2 \alpha} x^{2 \alpha}+2^{\alpha} t^{\alpha}(1-t)^{\alpha} x^{\alpha} y^{\alpha}+(1-t)^{2 \alpha} y^{2 \alpha}\right) \\
= & t^{\alpha} f(x)+(1-t)^{\alpha} f(y)-c^{\alpha} t^{\alpha} x^{2 \alpha}-c^{\alpha}(1-t)^{\alpha} y^{2 \alpha} \\
& +c^{\alpha} t^{2 \alpha} x^{2 \alpha}+2^{\alpha} c^{\alpha} t^{\alpha}(1-t)^{\alpha} x^{\alpha} y^{\alpha}+c^{\alpha}(1-t)^{2 \alpha} y^{2 \alpha} \\
= & t^{\alpha} f(x)+(1-t)^{\alpha} f(y)-c^{\alpha} t^{\alpha}(1-t)^{\alpha} x^{2 \alpha} \\
& +2^{\alpha} c^{\alpha} t^{\alpha}(1-t)^{\alpha} x^{\alpha} y^{\alpha}-c^{\alpha}(1-t)^{\alpha}\left(1^{\alpha}-(1-t)^{\alpha}\right) y^{2 \alpha} \\
= & t^{\alpha} f(x)+(1-t)^{\alpha} f(y)-c^{\alpha} t^{\alpha}(1-t)^{\alpha} x^{2 \alpha} \\
& +2^{\alpha} c^{\alpha} t^{\alpha}(1-t)^{\alpha} x^{\alpha} y^{\alpha}-c^{\alpha} t^{\alpha}(1-t)^{\alpha} y^{2 \alpha} \\
= & t^{\alpha} f(x)+(1-t)^{\alpha} f(y)-c^{\alpha} t^{\alpha}(1-t)^{\alpha}\left(x^{2 \alpha}-2^{\alpha} x^{\alpha} y^{\alpha}+y^{2 \alpha}\right) \\
= & t^{\alpha} f(x)+(1-t)^{\alpha} f(y)-c^{\alpha} t^{\alpha}(1-t)^{\alpha}(x-y)^{2 \alpha}
\end{aligned}
$$

for all $x, y \in I$ and $t \in[0,1]$. Thus, we have $f \in G S C_{\alpha}^{c}(I)$.

Theorem 2.9. Let $f: I \rightarrow \mathbf{R}^{\alpha}$. Then $f \in G S C_{\alpha}^{c}(I)$ if and only if the inequality

$$
\frac{f\left(x_{1}\right)-f\left(x_{2}\right)}{\left(x_{1}-x_{2}\right)^{\alpha}} \leq \frac{f\left(x_{3}\right)-f\left(x_{2}\right)}{\left(x_{3}-x_{2}\right)^{\alpha}}-c^{\alpha}\left(x_{3}-x_{1}\right)^{\alpha}
$$

holds, for any $x_{1}, x_{2}, x_{3} \in I$ with $x_{1}<x_{2}<x_{3}$.

Proof. Suppose that $f \in G S C_{\alpha}^{c}(I)$, then by Theorem 2.8, this is equivalent to saying that the function $g: I \rightarrow \mathbf{R}^{\alpha}$ defined by $g(x)=f(x)-c^{\alpha} x^{2 \alpha}$ is generalized convex. By [6, Theorem 8], the above is equivalent to the fact that the inequality

$$
\frac{f\left(x_{1}\right)-c^{\alpha} x_{1}^{2 \alpha}-f\left(x_{2}\right)+c^{\alpha} x_{2}^{2 \alpha}}{\left(x_{1}-x_{2}\right)^{\alpha}} \leq \frac{f\left(x_{3}\right)-c^{\alpha} x_{3}^{2 \alpha}-f\left(x_{2}\right)+c^{\alpha} x_{2}^{2 \alpha}}{\left(x_{3}-x_{2}\right)^{\alpha}}
$$


holds, for any $x_{1}, x_{2}, x_{3} \in I$ with $x_{1}<x_{2}<x_{3}$. Equivalently, we have

$$
\begin{aligned}
\frac{f\left(x_{1}\right)-f\left(x_{2}\right)}{\left(x_{1}-x_{2}\right)^{\alpha}} & \leq \frac{f\left(x_{3}\right)-f\left(x_{2}\right)}{\left(x_{3}-x_{2}\right)^{\alpha}}-c^{\alpha}\left[\frac{\left(x_{3}^{\alpha}-x_{2}^{\alpha}\right)\left(x_{3}^{\alpha}+x_{2}^{\alpha}\right)}{\left(x_{3}^{\alpha}-x_{2}^{\alpha}\right)}-\frac{\left(x_{2}^{\alpha}-x_{1}^{\alpha}\right)\left(x_{2}^{\alpha}+x_{1}^{\alpha}\right)}{\left(x_{2}^{\alpha}-x_{1}^{\alpha}\right)}\right] \\
& =\frac{f\left(x_{3}\right)-f\left(x_{2}\right)}{\left(x_{3}-x_{2}\right)^{\alpha}}-c^{\alpha}\left(x_{3}-x_{1}\right)^{\alpha} .
\end{aligned}
$$

Remark 2.10. Proceeding as in the proof of Theorem 2.4, it is shown that $f \in G S C_{\alpha}^{c}(I)$ if and only if

$$
\begin{aligned}
\frac{f\left(x_{2}\right)-f\left(x_{1}\right)}{\left(x_{2}-x_{1}\right)^{\alpha}}-c^{\alpha}\left(x_{3}-x_{1}\right)^{\alpha} & \leq \frac{f\left(x_{2}\right)-f\left(x_{1}\right)}{\left(x_{2}-x_{1}\right)^{\alpha}} \leq \frac{f\left(x_{3}\right)-f\left(x_{1}\right)}{\left(x_{3}-x_{1}\right)^{\alpha}}-c^{\alpha}\left(x_{3}-x_{2}\right)^{\alpha} \\
& \leq \frac{f\left(x_{3}\right)-f\left(x_{1}\right)}{\left(x_{3}-x_{1}\right)^{\alpha}} \leq \frac{f\left(x_{3}\right)-f\left(x_{2}\right)}{\left(x_{3}-x_{2}\right)^{\alpha}}-c^{\alpha}\left(x_{2}-x_{1}\right)^{\alpha} \\
& \leq \frac{f\left(x_{3}\right)-f\left(x_{2}\right)}{\left(x_{3}-x_{2}\right)^{\alpha}},
\end{aligned}
$$

for any $x_{1}, x_{2}, x_{3} \in I$ with $x_{1}<x_{2}<x_{3}$.

Theorem 2.11 (generalized Hermite-Hadamard-type inequality). If $f \in I_{x}^{(\alpha)}[a, b]$ and $f \in G S C_{\alpha}^{c}[a, b]$, then

$$
\begin{aligned}
f\left(\frac{a+b}{2}\right)-c^{\alpha}\left(\frac{a+b}{2}\right)^{2 \alpha} & \leq \frac{\Gamma(1+\alpha)}{(b-a)^{\alpha}}\left[{ }_{a} I_{b}^{(\alpha)} f(x)-c^{\alpha} \frac{\Gamma(1+2 \alpha)}{\Gamma(1+3 \alpha)}\left(b^{3 \alpha}-a^{3 \alpha}\right)\right] \\
& \leq \frac{f(a)+f(b)}{2^{\alpha}}-c^{\alpha}\left(\frac{a^{2 \alpha}+b^{2 \alpha}}{2^{\alpha}}\right) .
\end{aligned}
$$

Proof. Suppose that $f \in I_{x}^{(\alpha)}[a, b]$ and $f \in G S C_{\alpha}^{c}[a, b]$, then by Theorem 2.8 , this is equivalent to saying that the function $g:[a, b] \rightarrow \mathbf{R}^{\alpha}$ defined by $g(x)=f(x)-c^{\alpha} x^{2 \alpha}$ is generalized convex. By [6, Theorem 14], the above implies that the generalized Hermite-Hadamard inequality holds for $g$; i.e.

$$
g\left(\frac{a+b}{2}\right) \leq \frac{\Gamma(1+\alpha)}{(b-a)^{\alpha}}{ }_{a} I_{b}^{(\alpha)} g(x) \leq \frac{g(a)+g(b)}{2^{\alpha}} .
$$

Equivalently, we have 


$$
\begin{aligned}
f\left(\frac{a+b}{2}\right)-c^{\alpha}\left(\frac{a+b}{2}\right)^{2 \alpha} & \leq \frac{\Gamma(1+\alpha)}{(b-a)^{\alpha}}{ }_{a} I_{b}^{(\alpha)}\left[f(x)-c^{\alpha} x^{2 \alpha}\right] \\
& \leq \frac{f(a)+f(b)}{2^{\alpha}}-c^{\alpha}\left(\frac{a^{2 \alpha}+b^{2 \alpha}}{2^{\alpha}}\right)
\end{aligned}
$$

Consequently,

$$
\begin{aligned}
f\left(\frac{a+b}{2}\right)-c^{\alpha}\left(\frac{a+b}{2}\right)^{2 \alpha} & \leq \frac{\Gamma(1+\alpha)}{(b-a)^{\alpha}}{ }_{a} I_{b}^{(\alpha)} f(x)-c^{\alpha} \frac{\Gamma(1+\alpha) \Gamma(1+2 \alpha)}{\Gamma(1+3 \alpha)} \frac{\left(b^{3 \alpha}-a^{3 \alpha}\right)}{(b-a)^{\alpha}} \\
& \leq \frac{f(a)+f(b)}{2^{\alpha}}-c^{\alpha}\left(\frac{a^{2 \alpha}+b^{2 \alpha}}{2^{\alpha}}\right) .
\end{aligned}
$$

Thus, we have

$$
\begin{aligned}
f\left(\frac{a+b}{2}\right)-c^{\alpha}\left(\frac{a+b}{2}\right)^{2 \alpha} & \leq \frac{\Gamma(1+\alpha)}{(b-a)^{\alpha}}\left[{ }_{a} I_{b}^{(\alpha)} f(x)-c^{\alpha} \frac{\Gamma(1+2 \alpha)}{\Gamma(1+3 \alpha)}\left(b^{3 \alpha}-a^{3 \alpha}\right)\right] \\
& \leq \frac{f(a)+f(b)}{2^{\alpha}}-c^{\alpha}\left(\frac{a^{2 \alpha}+b^{2 \alpha}}{2^{\alpha}}\right) .
\end{aligned}
$$

\section{References}

[1] A. Azócar, J. Giménez, K. Nikodem, and J. L. Sánchez, "On strongly midconvex functions", Opuscula mathematica, vol. 31, no. 1, pp. 15-26, 2011, doi:10.7494/OpMath.2011.31.1.15.

[2] G.-S. Chen, "A generalized Young inequality and some new results on fractal space", Jul. 2011. arXiv: 1107.5222v1

[3] K. M. Kolwankar and A. D. Gangal, "Fractional differentiability of nowhere differentiable functions and dimensions", Chaos, vol. 6, no. 4, pp. 505-513, 1996, doi: 10.1063/1.166197. 
[4] T. Lara, N. Merentes, R. Quintero and E. Rosales, "On strongly mconvex functions", Mathematica aeterna, vol. 5, no. 3, pp. 521-535, 2015. [On line]. Available: https://bit.ly/2G6kHpF

[5] N. Merentes and K. Nikodem, "Remarks on strongly convex functions", Aequationes mathematicae, vol. 80, no. 1-2, pp. 193-199. 2010, doi: 10.1007/s00010-010-0043-0.

[6] H. Mo, X. Sui and D. Yu, "Generalized convex functions on fractal sets and two related inequalities", Abstract and applied analysis, Art. ID 636751, 2014, doi: 10.1155/2014/636751.

[7] L. Montrucchio, "Lipschitz policy functions for strongly concave optimization problems", Journal of mathematical economics, vol. 16, no. 3, pp. 259-273, 1987, doi: 10.1016/0304-4068(87)90012-7.

[8] B. T. Polyak, "Existence theorems and convergence of minimizing sequences in extremum problems with restrictions", Soviet mathematics. Doklady, vol. 166, no. 2, pp. 72-75, 1966.[On line]. Available: https://bit.ly/2v9BrtX

[9] A. W. Roberts and D. E. Varberg, "Convex functions", New York, NY: Academic Press, 1973.

[10] W. Sun, "Generalized harmonically convex functions on fractal sets and related Hermite-Hadamard type inequalities", Journal of nonlinear sciences and applications, vol. 10, no. 11, pp. 5869-5880, 2017, doi: 10.22436/jnsa.010.11.24.

[11] X.-J. Yang, "Advanced local fractional calculus and its applications", New York, NY: World Science, 2012.

[12] X.-J. Yang, "Expression of generalized newton iteration method via generalized local fractional Taylor series", Jun. 2012. arXiv:1106.2780v2 